\title{
Multi-Agent System-Based Microgrid Operation Strategy for Demand Response
}

\author{
Hee-Jun Cha ${ }^{1}$, Dong-Jun Won ${ }^{1, *}$, Sang-Hyuk Kim ${ }^{2}$, Il-Yop Chung ${ }^{2}$ and Byung-Moon Han ${ }^{3}$ \\ Received: 14 September 2015; Accepted: 14 December 2015; Published: 18 December 2015 \\ Academic Editor: Neville R. Watson \\ 1 Department of Electrical Engineering, Inha University, 100, Inha-ro, Nam-gu, Incheon 402-751, Korea; \\ chj119119@gmail.com \\ 2 School of Electrical Engineering, Kookmin University, Jeongneung-ro, Seongbuk-gu, Seoul 136-702, Korea; \\ dungate@naver.com (S.-H.K.); chung@kookmin.ac.kr (I.-Y.C.) \\ 3 Department of Electrical Engineering, Myongji University, 116, Myongji-ro, Cheoin-gu, Yongin-si, \\ Gyeonggi-do 449-728, Korea; erichan@mju.ac.kr \\ * Correspondence: djwon@inha.ac.kr; Tel.: +82-32-860-7404; Fax: +82-32-863-5822
}

\begin{abstract}
The microgrid and demand response (DR) are important technologies for future power grids. Among the variety of microgrid operations, the multi-agent system (MAS) has attracted considerable attention. In a microgrid with MAS, the agents installed on the microgrid components operate optimally by communicating with each other. This paper proposes an operation algorithm for the individual agents of a test microgrid that consists of a battery energy storage system (BESS) and an intelligent load. A microgrid central controller to manage the microgrid can exchange information with each agent. The BESS agent performs scheduling for maximum benefit in response to the electricity price and BESS state of charge (SOC) through a fuzzy system. The intelligent load agent assumes that the industrial load performs scheduling for maximum benefit by calculating the hourly production cost. The agent operation algorithm includes a scheduling algorithm using day-ahead pricing in the DR program and a real-time operation algorithm for emergency situations using emergency demand response (EDR). The proposed algorithm and operation strategy were implemented both by a hardware-in-the-loop simulation test using OPAL-RT and an actual hardware test by connecting a new distribution simulator.
\end{abstract}

Keywords: microgrid; demand response; multi-agent system; battery energy storage system; intelligent load; fuzzy system; hardware-in-the-loop simulation

\section{Introduction}

A microgrid is a small power system that includes distributed generation (DG), an energy storage system, and a controllable load [1,2]. In general, a microgrid operates optimally with the microgrid central controller (MGCC), which can control DG and the controllable load in addition to monitoring the microgrid system [3]. Typically, the microgrid can be operated in islanded mode or grid-connected mode [4]. In the islanded mode, the MGCC has to ensure power balance and control of voltage and frequency in the microgrid, but in the grid-connected mode, the MGCC can operate more economically because the utility grid is responsible for solving problems such as voltage control and frequency oscillation in the microgrid.

Intelligent agents can determine their performance autonomously by recognizing the surrounding environment and communicating with other agents. On the basis of these features, the microgrid can be operated using a number of agents. Recently, a microgrid operation technique through multi-agent system (MAS) has been studied [5,6]. Similar to the central control method of a traditional power system, a top-level agent or energy management system ensures the optimal 
operation of a microgrid through hierarchical information exchange between agents with specific purposes [6]. Each agent has its own operation algorithm. Therefore, this is a distributed control method involving cooperation or competition between agents for optimal operation [7].

In recent power systems, demand response (DR) is also important. DR technology can solve problems such as peak reduction, expansion of facilities, and transmission congestion. The DR changes the electricity price, and therefore consumers can adjust their load to reduce the price. The DR program can be classified as a price-based DR program and an incentive-based DR program. Price-based DR has time-of-use, day-ahead pricing (DAP), critical peak pricing, and real-time pricing (RTP). Incentive-based DR has emergency demand response (EDR), direct load control, and capacity market program (CMP) [8].

In the centralized control method of the microgrid, the MGCC aggregates all the information for optimal operation using a genetic algorithm (GA) [9], particle swarm optimization, and dynamic programming (DP) [10]. In this method, the DG and the battery energy storage system (BESS) output are determined in accordance with the MGCC optimization results. This optimization method is suitable for the best operation, but the calculation time is long, and all data are required at the MGCC. However, in the distributed control method, the MGCC sends the electricity price and state of the grid to agents for distribution control. Its role is minimal and confined to sending signals pertaining to the stability of the microgrid. In the other method of microgrid control, the system includes an agent platform for the agent's trade [7].

This study examined the operation strategy of a microgrid that consists of a BESS and an intelligent load using MAS. The MGCC can monitor the output through each agent and send data for each distributed independent operation. This study examined the economic operation algorithm using a fuzzy system for the BESS that receives the minimum information from the MGCC. The operation algorithm of an intelligent load agent, with the load assumed to be an industrial load, was also studied for maximum benefit by calculating the hourly production cost [11]. The two agents schedule using DAP and perform a real-time operation when EDR occurs. As the microgrid is connected to the grid, the microgrid maintains stability at the point of common coupling (PCC). Therefore, the MGCC in this study ensures individual agent operation. However, if the microgrid becomes unstable, the MGCC intervenes in the agent's operation to restore microgrid stability.

This paper is organized as follows: Section 2 examines each agent operation algorithm and the MGCC operation algorithm. In Section 3, the proposed algorithm is analyzed with different simulation scenarios through hardware-in-the-loop system (HILS) test by constructing the agents and the MGCC. Section 4 describes the hardware test performed with a new distribution simulator for comparison with the HILS test results in Section 3. Finally, Section 5 presents the conclusions of the study.

\section{Operation Algorithms of Individual Agents}

In this study, a test microgrid is constructed with a BESS, an intelligent load, and an MGCC that can communicate with two agents as shown in Figure 1. Each agent executes operation algorithms independently without affecting each other. Because the test microgrid is assumed to be for a commercial purpose, the owner of all components may be different. Thus, for independence and stability of the system, the MGCC receives data from the grid-side energy management system (EMS) and sends data and signals to two agents, each of which has two operation algorithms: a day-ahead scheduling algorithm and a real-time algorithm in accordance with the EDR signal. The price data for scheduling is used in the DAP, which is one of the DR programs. On any day, the DAP program notifies the price of $24 \mathrm{~h}$ electricity for the next day. Similarly, EDR is included in the DR program.

The BESS agent has the objective of maximizing the economic benefit from the energy arbitrage [12]. In addition, because it maintains a certain state-of-charge (SOC) range, the agent can contribute to power system stability by discharging when an emergency situation occurs. The intelligent load agent that assumes an industrial load performs production scheduling through 
DAP to minimize the electricity price. The agent can compare the sales benefit and incentive for participation in EDR and determine whether to participate in EDR. The detailed algorithm is described as follows.

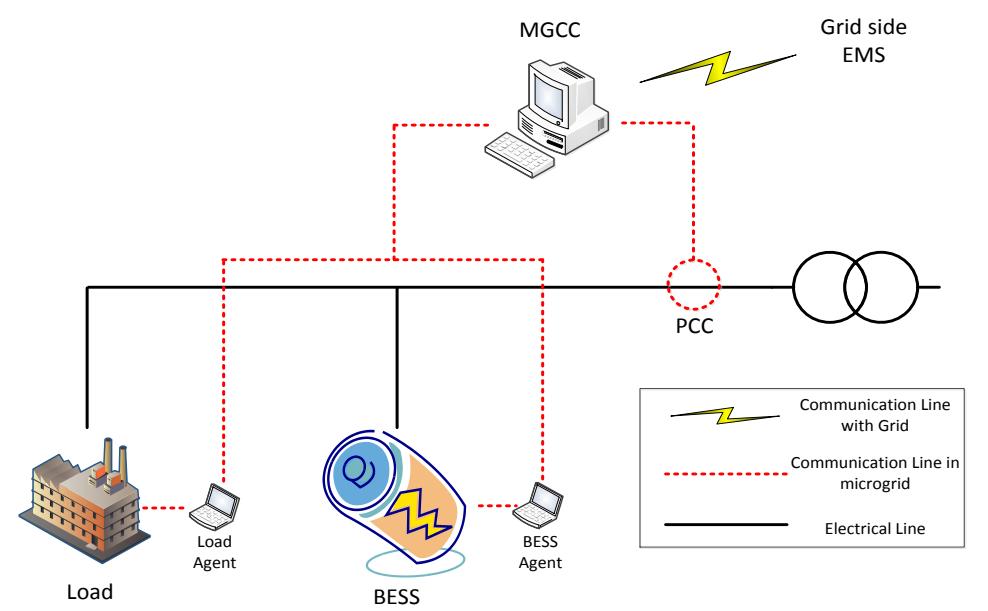

Figure 1. Configuration of microgrid in this paper.

\subsection{Algorithm for BESS Agent}

The BESS is used for frequency regulation, system reserve, demand-side management capacity, and augmentation of renewable energy resources [13]. In this study, the scheduling algorithm of the BESS is designed using a fuzzy expert system that can obtain an appropriate margin during the day and a real-time operation algorithm is responsible for handling emergency situations. The fuzzy expert system utilizes fuzzy logic to represent ambiguous scenarios instead of Boolean logic [14].

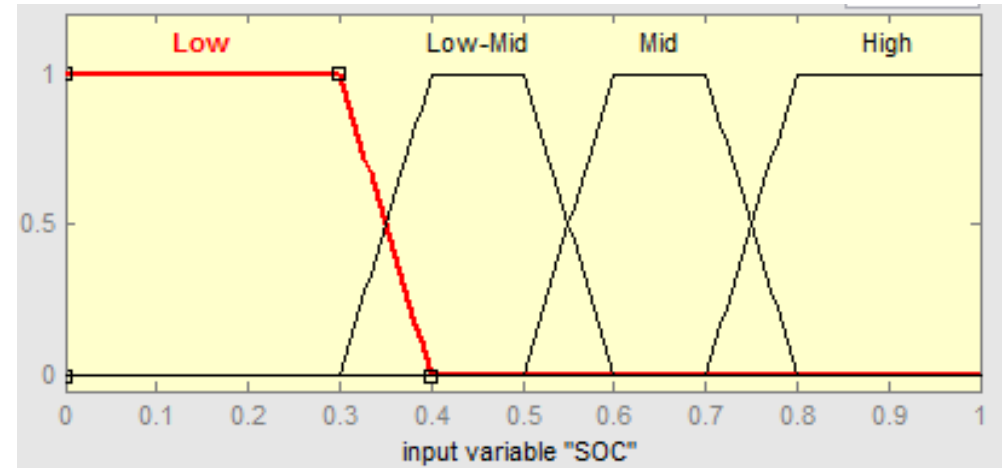

(a)

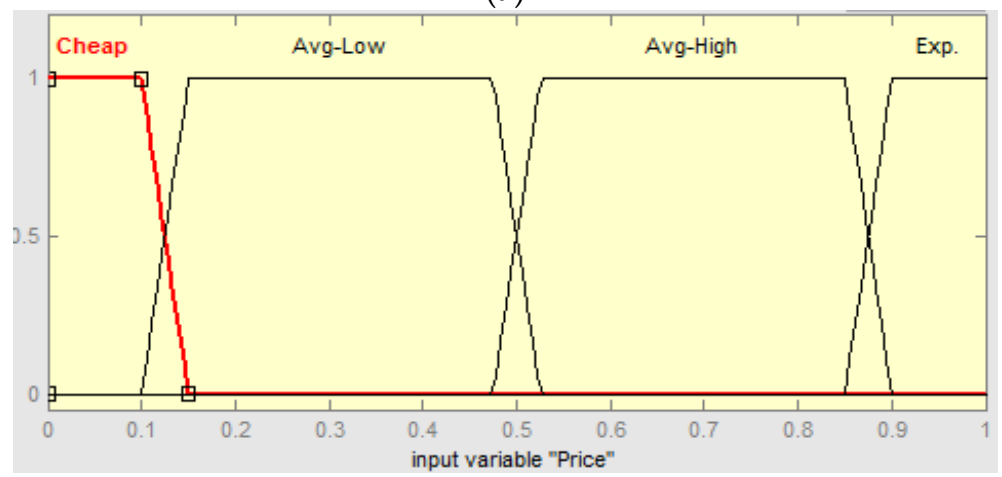

(b)

Figure 2. Cont. 


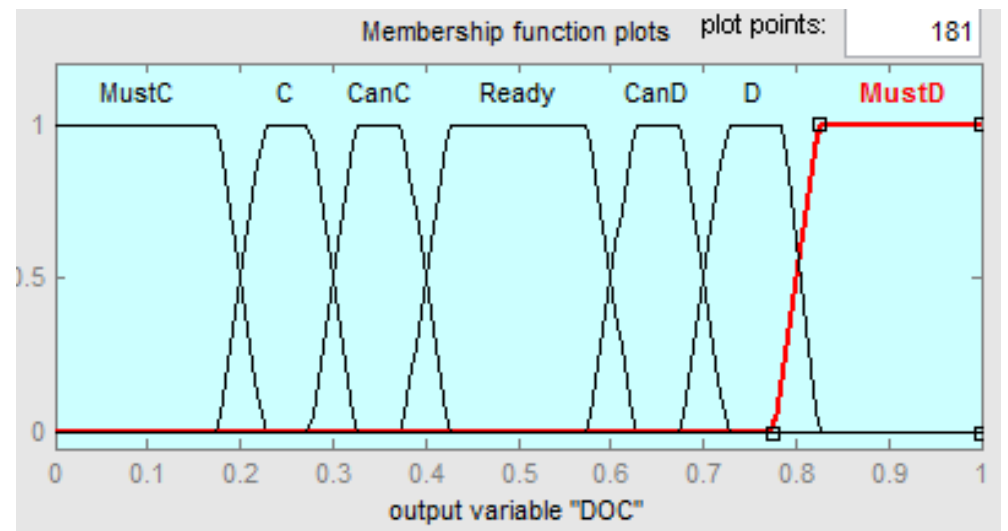

(c)

Figure 2. Fuzzy membership function (a) state of charge (SOC), (b) electricity price, (c) decision of charge.

In this study, the scheduling algorithm was designed to determine if it is charging or discharging using the fuzzy system as the input membership functions of the BESS SOC and hourly DAP. Figure 2 shows a membership function of the fuzzy system applied in this study. In the step-by-step descriptions, the BESS's SOC and the electricity price are determined by a preset fuzzy input membership function. The output membership function is determined by the SOC and the electricity price membership function through the fuzzy rule. Table 1 represents the fuzzy rule in this algorithm. All cases were evaluated and combined. Finally, the charge/discharge value was determined by the scalar value whose aggregated output membership function is defuzzification by the centroid method.

Table 1. Fuzzy rule.

\begin{tabular}{ccccc}
\hline SOC/Price & Cheap & Avg-Low & Avg-High & Expensive \\
\hline Low & Must Charge & Charge & Can Charge & Ready \\
Low-Mid & Charge & Can Charge & Ready & Can Discharge \\
Mid & Can Charge & Ready & Can Discharge & Discharge \\
High & Ready & Can Discharge & Discharge & Must Discharge \\
\hline
\end{tabular}

Figure 3 shows a flowchart of the BESS scheduling algorithm. The BESS has an agent that receives the electricity price from the MGCC. The agent determines the hourly BESS output using the fuzzy system with the hourly SOC and electricity price. In the scheduling algorithm, the BESS has a driving range of $30 \%$ to $100 \%$ of the SOC. When the calculated SOC is greater or lower than the operating range, the agent determines the maximum value within the SOC constraints. The defuzzification value from the fuzzy system determines the BESS power output. Depending on this output, it is possible to perform BESS scheduling over a $24 \mathrm{~h}$ period by repeatedly calculating the SOC of the next time. Figure 4 presents a flowchart of the real-time BESS operation algorithm. When the agent receives an emergency signal from the MGCC, the BESS discharges until an SOC of $20 \%$ is reached. After the EDR periods, the BESS performs rescheduling with the scheduling algorithm for the remaining time.

\subsection{Algorithm for Intelligent Load Agent}

It should be noted that the intelligent load in this study is assumed to be an industrial load for the manufacturing industry. An intelligent load consists of a controllable load that is controlled by the time, and a fixed load that is maintained at all times for the operation of a factory. In this paper, an intelligent load scheduling algorithm for DR is proposed considering the labor costs and 
the electricity price of the different time period from the authors' previous study [11]. In general, when a product is produced, its costs can be classified as the raw material costs, labor cost, electricity price, and other costs (transmission expense and external effect, etc.). The raw material cost and other costs are fixed costs. Therefore, the labor cost and electricity price in each time period are important variables. Thus, the hourly production costs calculated by Equations (1) and (2) are used in the scheduling algorithm for the maximum benefit per day.

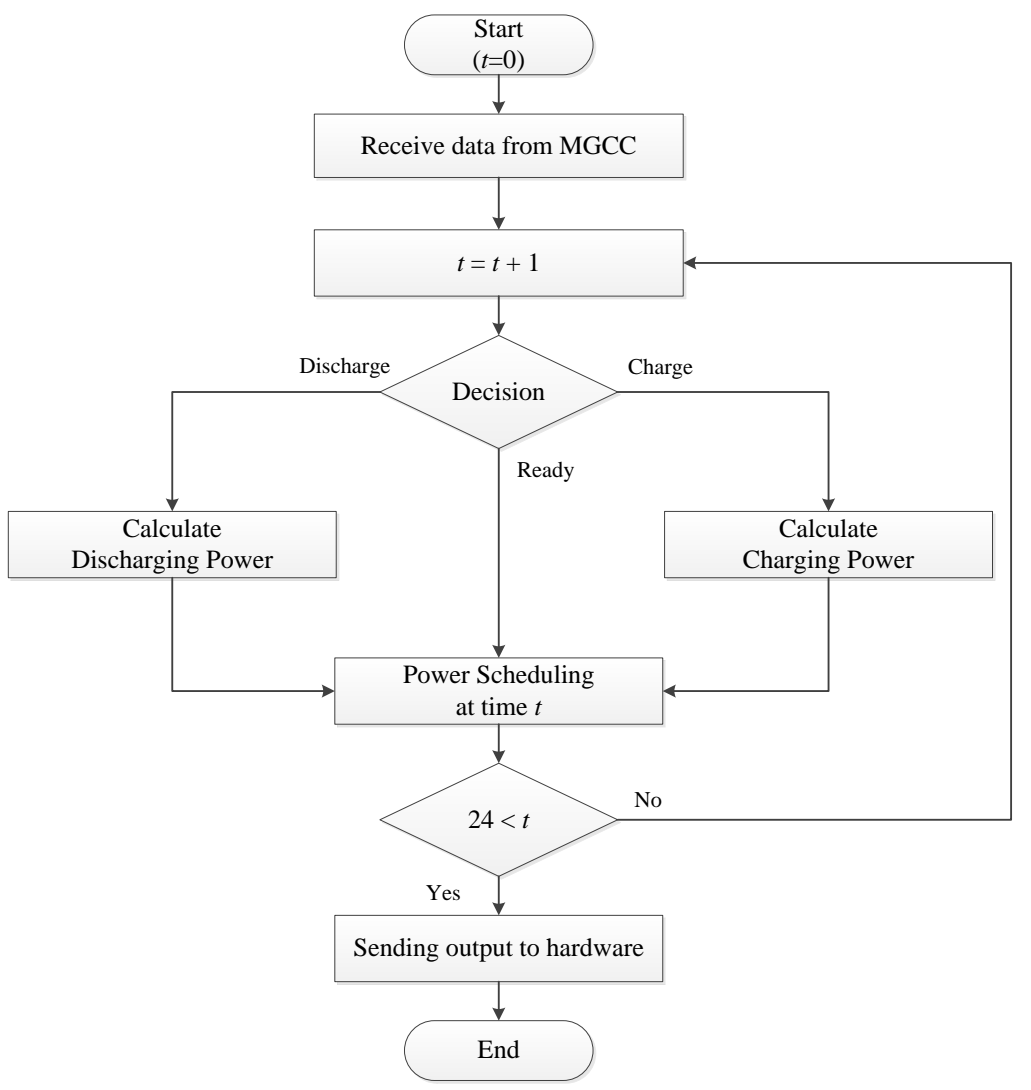

Figure 3. Flow chart of battery energy storage system (BESS) scheduling algorithm.

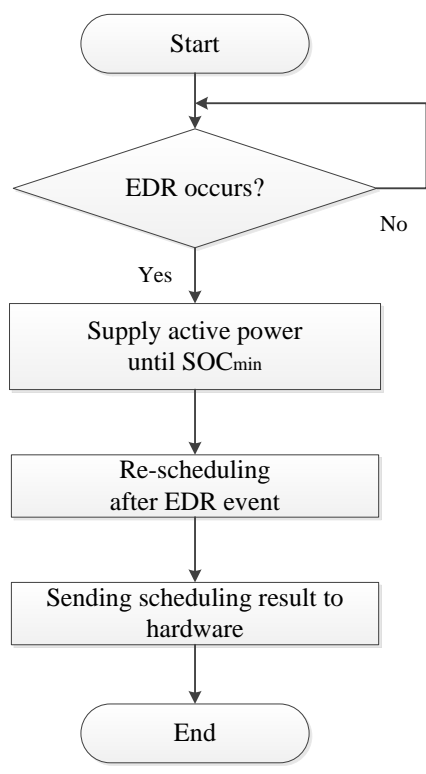

Figure 4. Flow chart of BESS real-time algorithm. 
At each time, this can be expressed by the formula as follows Equations (1) and (2):

$$
\begin{gathered}
\operatorname{Cost}_{i}(t)=C_{P}+\alpha \times C_{\text {initial }}(t)+\varepsilon \times C_{P E}(t) \\
\text { Benefit }_{\text {sell }}(t)=\text { Price }_{\text {sell }}-\text { Cost }_{i}(t)
\end{gathered}
$$

where:

$\operatorname{Cost}_{i}(t)$ : Product cost of each load type at time $t$;

$C_{P}$ : Raw material cost;

$\alpha$ : Electricity price rate per product;

$C_{\text {initial }}(t)$ : Electricity price at time $t$;

$\varepsilon$ : Labor cost rate per product;

$C_{P E}(\mathrm{t})$ : Labor cost at time $t$;

Price $_{\text {sell }}(t)$ : Product's selling price;

Benefit $t_{\text {sell }}$ : Benefit between product price and cost.

Equation (1) represents the cost of the product, and Equation (2) represents the sale benefit per product. Finally, labor costs are classified as normal working hours, overtime working hours, and night working hours as listed in Table 2.

Table 2. Labor cost rate at each time period.

\begin{tabular}{cccc}
\hline \multirow{2}{*}{ Time } & AM. 8-PM. 6 & PM. 6-PM. 10 & Others \\
\cline { 2 - 4 } & Normal Work & Extended Work & Night Work \\
\hline Labor cost rate & 1 & 1.5 & 2 \\
\hline
\end{tabular}

Figure 5 presents a flowchart for the intelligent load scheduling algorithm. The intelligent load agent received the electric price data of each time interval from the MGCC. The intelligent load agent calculates the production costs in Equation (1) and sorts them in ascending order. The intelligent load agent then uses the maximum power in order from the lowest cost of time. The remainder of the production volume after using the maximum power is used in the next time with low cost. When the scheduling algorithm is complete, the intelligent load agent sends data to the load hardware in chronological order.

Figure 6 presents the flowchart of an intelligent load real-time operating algorithm. Initially, the daily schedule of the load is determined by the scheduling algorithm. The load agent verifies whether the signal is received from the MGCC before EDR occurs. The EDR signal has two data sets: (1) time period of EDR and (2) incentive by participating in EDR:

$$
I(\text { EDR time })>\left[\left(P_{\text {con }}(\text { EDR time }) \div a\right) \times \text { Benefit }_{\text {sell }}(t)\right] \div P_{\text {Load }}(t)
$$

where:

$I(t)$ : Incentive at time $t$;

$P_{\text {con }}(t)$ : Controllable Power at time $t$;

$P_{\text {Load }}(t)$ : Load Power at time $t$.

In Equation (3), $P_{c o n}(t)$ denotes the controllable power for manufacturing of products, and $P_{\text {Load }}(t)$ denotes the entire load power, which includes the controllable power and the fixed load for operating the factory. When the EDR signal is received at the load agent, the agent confirms Equation (3). If the equation is satisfied, the agent decides to participate in EDR. If the right-hand term is larger than the left-hand term in Equation (3), the agent retains the original scheduling. If the load participates in EDR, the agent reschedules for the elapsed time period of EDR in accordance 
with the scheduling algorithm. On the other hand, if the load does not participate in EDR, the load agent is affected by the scheduling algorithm. Here, it assumes that the power requirement at EDR is greater than the load. Therefore, all controllable loads except for the fixed load should be reduced in that time period.

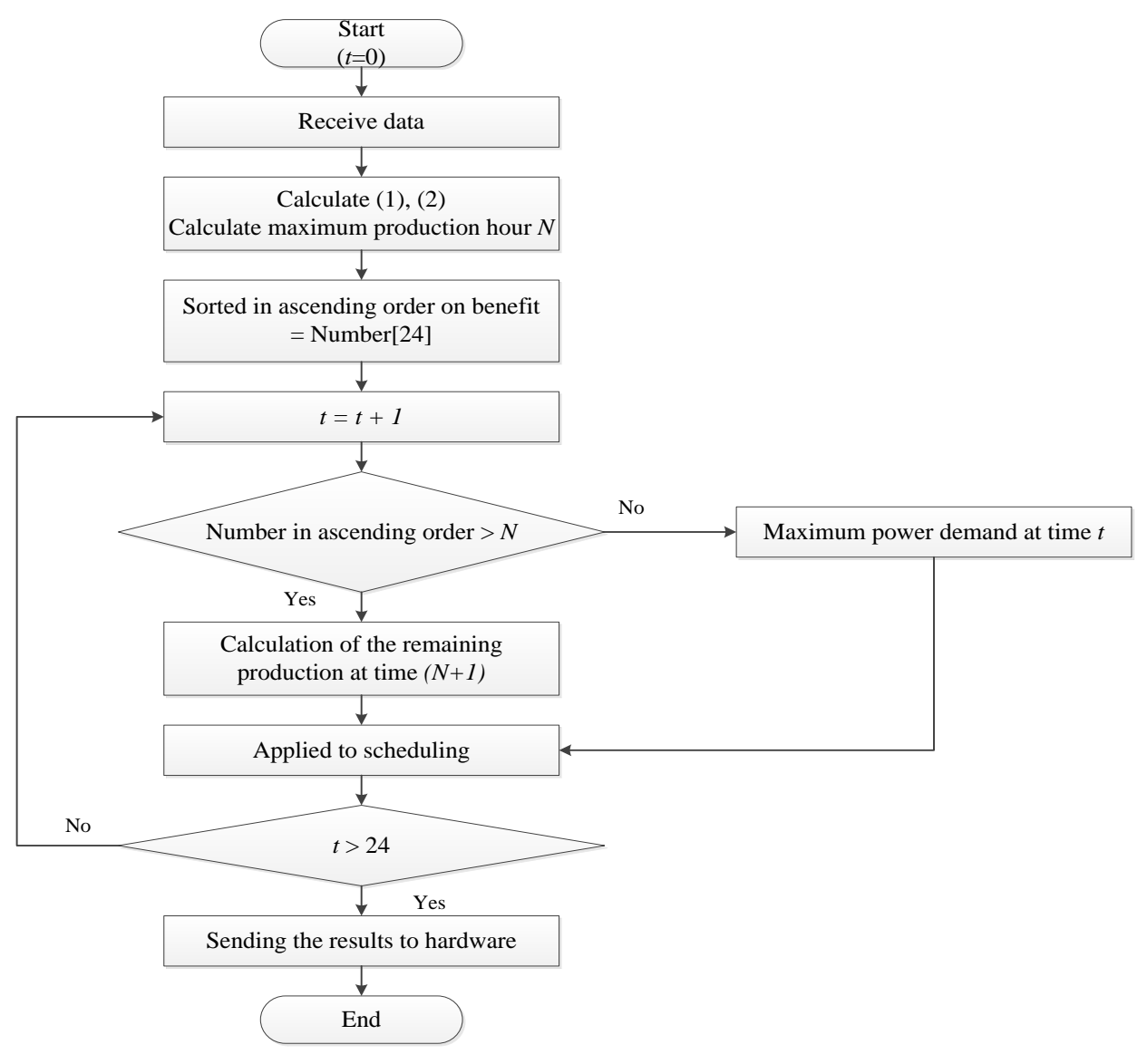

Figure 5. Flow chart of intelligent load scheduling algorithm.

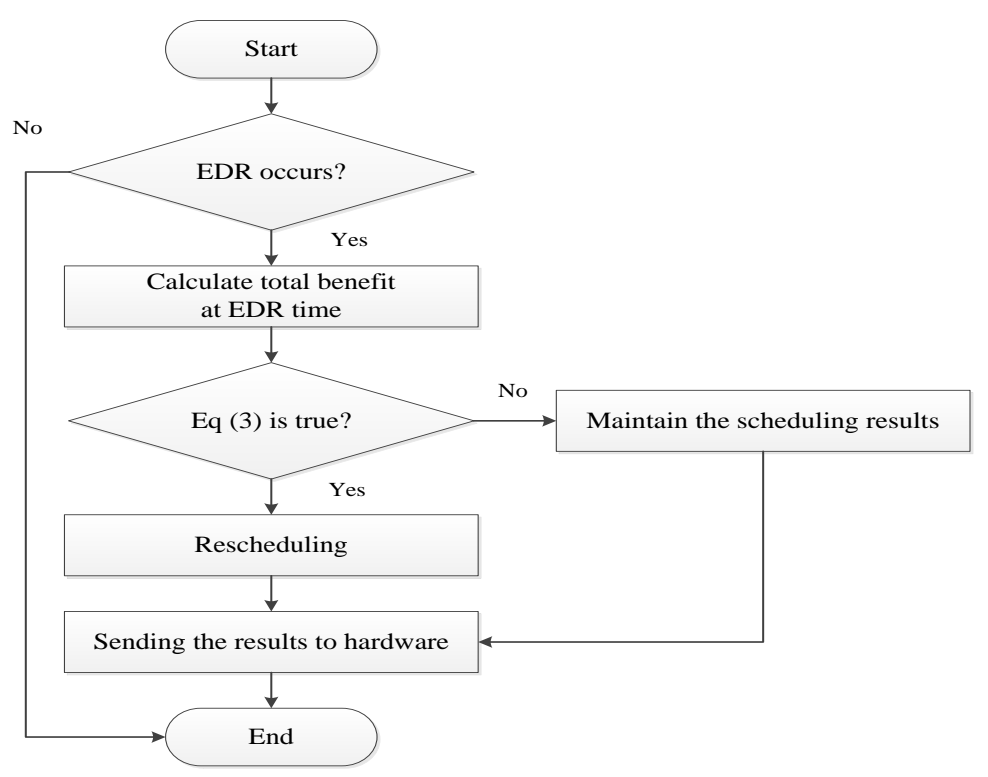

Figure 6. Flow chart of intelligent load real-time algorithm. 


\subsection{Operation Algorithm for MGCC}

Figure 7 is the MGCC management algorithm considering the overall system stability. In general, the MGCC receives data (electricity price, emergency signal) from the upper EMS and sends this data and signal to lower agents. Further, the MGCC can monitor the power and control by sending any signal to the agents. In the grid-connected microgrid, however, problems such as the capacity of a transformer, transmission/distribution cable, and capacity of the PCC can occur. Considering these problems, the MGCC receives the expected charging power from the BESS agent, and if the total power in the microgrid exceeds the allowable value at the PCC, the MGCC sends a charging delay signal to the BESS agent. If not, the MGCC does not send a delay signal. The MGCC considers the power in the microgrid and the PCC capacity. Therefore, this system can be applied without other system changes if another DG or load is added to the system and the communication has been transmitted.

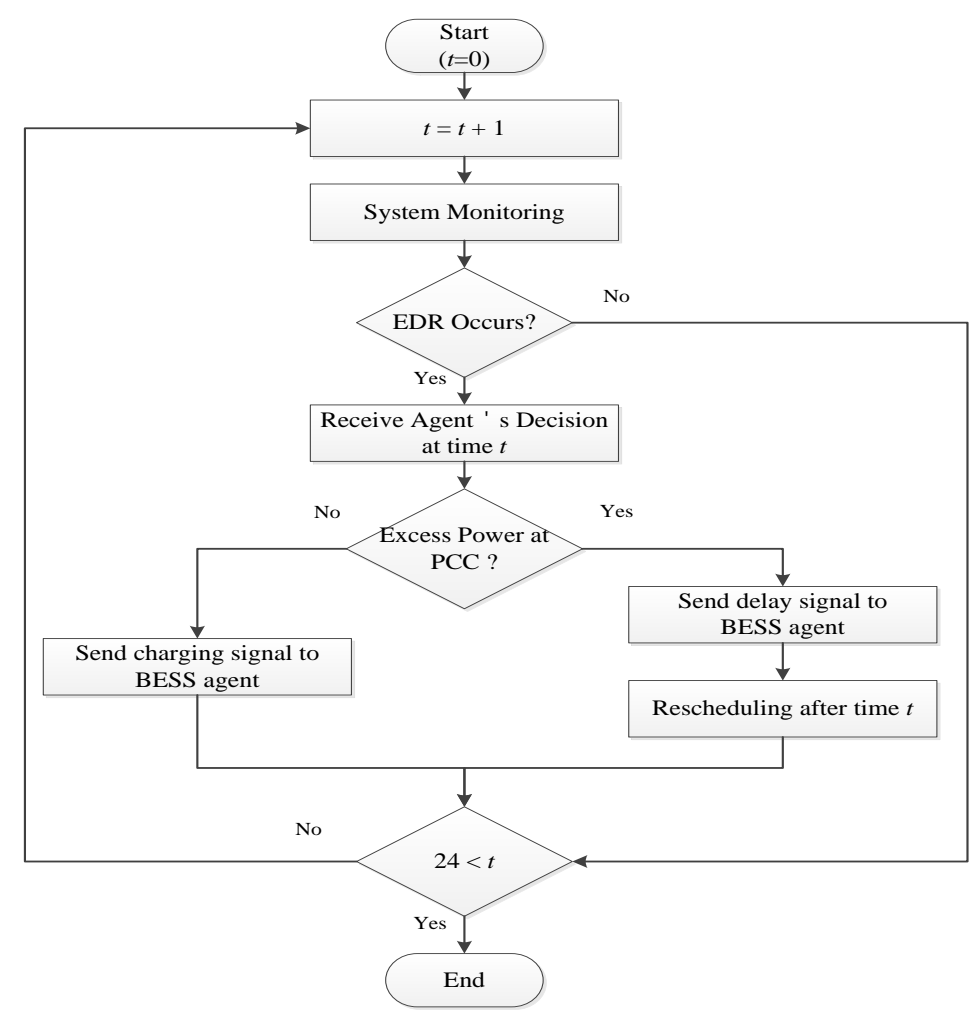

Figure 7. Flow chart of microgrid central controller (MGCC) operation algorithm.

\section{Simulation Results}

Figure 8 shows the construction of the HILS test system with the MGCC program and agents. In this study, the MGCC program is used for monitoring and managing this system, and the agents of the BESS and load were constructed to verify the performance of the proposed algorithm and simulate the microgrid operation [15]. The following describes each component of the system. Before connecting to the hardware, OPAL-RT was used for the HILS to test the communication and operation between the MGCC and the agent.

\subsection{MGCC Program}

The MGCC program was constructed using Visual Basic 6.0 and communicates with each agent using Zigbee. First, when each agent's ID is sent to the MGCC and registered, it follows the monitoring and control of the agent's state. As shown in Figure 8, the MGCC program shows the status and mode of the microgrid operation and sends a scheduling and emergency signal to agents. 
The scheduling and emergency signal sent by the MGCC contains information on the hourly price, emergency predictive time, and incentive.

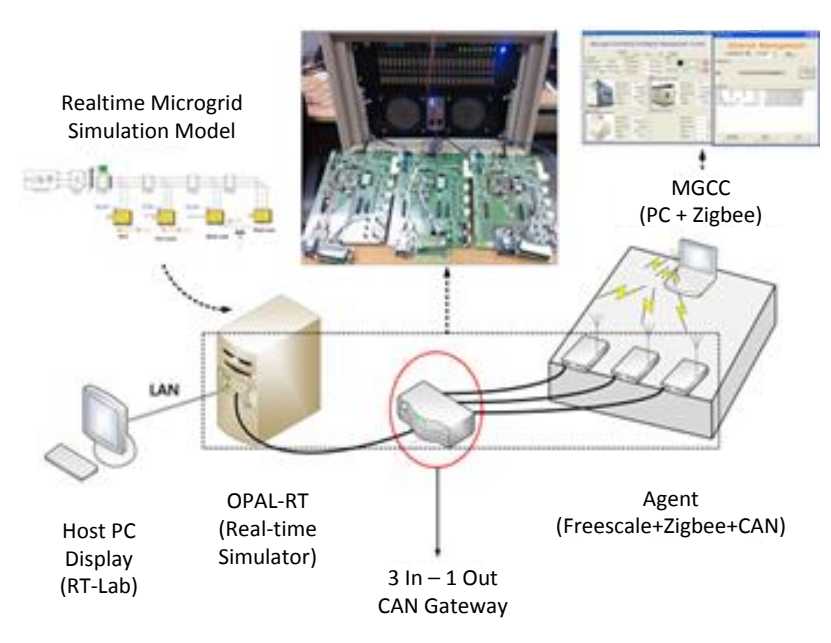

Figure 8. Hardware-in-the-loop (HILS) test configuration.

\subsection{Agents}

In this study, the agent consisted of a FreeScale board (EVB9S12XDP512). The agent performed each operation algorithm by receiving the signal containing a variety of information from the MGCC. The agent was connected by hardware using a CAN bus. Therefore, each agent could monitor and control the power output of the hardware connected to the agent.

\subsection{Case Study}

Table 3 shows scenarios for the verification of each agent's algorithm. The RTP electricity price in this study was applied by referring to the Illinois state RTP program in the United States. As mentioned earlier, the test microgrid consisted of the BESS and the intelligent load. The BESS in all scenarios was set to an initial SOC of $40 \%$, a rate power of $1 \mathrm{~kW}$, and a rate capacity of $3 \mathrm{~kW} / \mathrm{h}$. With an intelligent load, it was assigned a minimum maintenance load of $0.6 \mathrm{~kW}$ and a controllable load of $1.8 \mathrm{~kW}$. In all scenarios, the hourly maximum production volume was 11 , and the daily maximum production volume was 126 . In practice, the load and the BESS rate power were higher than the setting value. On the other hand, the hardware testing in laboratory condition was set as described earlier.

Table 3. Scenario for the proposed algorithm.

\begin{tabular}{|c|c|c|c|c|}
\hline Number & Common Condition & Test Type & Scenario & Measure \& Evaluation \\
\hline 1 & \multirow{3}{*}{$\begin{array}{l}\text { (1) BESS, Load } \\
\text { (2) Fixed load }=0.6 \mathrm{~kW} \\
\text { (3) BESS initial } \\
\text { SOC }=40 \% \\
\text { (4) Production } \\
\text { volume }=11 / \mathrm{h}, 26 / \text { day }\end{array}$} & Scheduling & (1) DAP in summer & BESS, Load output moment \\
\hline 2 & & \multirow[b]{2}{*}{ Real-time } & $\begin{array}{l}\text { (1) DAP in summer } \\
\text { (2) EDR in } 13-15 \mathrm{~h} \\
\text { (3) No delay signal from MGCC }\end{array}$ & $\begin{array}{l}\text { (1) BESS, Load output moment } \\
\text { (2) Response to the EDR signal } \\
\text { (3) delay or not }\end{array}$ \\
\hline 3 & & & $\begin{array}{l}\text { (1) DAP in summer } \\
\text { (2) EDR in } 13-15 \mathrm{~h} \\
\text { (3) Adapt delay signal (Maximum } \\
\text { power at PCC }=2.5 \mathrm{~kW} \text { ) }\end{array}$ & $\begin{array}{l}\text { (1) BESS, Load output moment } \\
\text { (2) Response to the EDR signal } \\
\text { (3) delay or not }\end{array}$ \\
\hline
\end{tabular}




\subsubsection{Scenario 1: DAP of Summer}

Figure 9 shows the HILS test result in scenario 1. The RTP in summer is similar to the load curve, in which the load is higher in the afternoon than in the morning. Figure 8 shows the electricity price production cost, load active power, BESS active power, and BESS SOC from the top to the bottom. Each agent starting from (1) makes the power output reference that is conducted by the operation algorithm in time order. The hourly production cost of the products can be derived from the electric price from the load agent. Therefore, it is possible to perform load scheduling line with the hourly cost of production for maximum benefit. Accordingly, the load can be confirmed by the lowest production cost to the maximum productivity. Since product cost is affected by the labor cost, the industrial load is operated during daytime with low labor cost. In the BESS, the agent charges at dawn at a low electricity price and initial SOC and discharges at 7 and $10 \mathrm{~h}$ at the comparatively high electricity price and SOC. The 7 and $10 \mathrm{~h}$ are not the most expensive time period of electricity price. However, since the SOC that is one of input variables of the fuzzy system is enough high, the BESS can discharge. The SOC is relatively low at time (2) but the BESS discharge because the electricity price is in the most expensive time period. The BESS charges again because the SOC is too low at time 18 . Scenario 1 is the base scenario that is compared to the other scenarios. The base scenario can be show the following. (1) The load agent is affected by labor cost as compared to the electricity price; (2) The BESS agent performs charge/discharge for maintaining a certain SOC range; (3) As shown in Figure 9, the two agents do not interfere with each other and the two agents consumed power at $10 \mathrm{~h}$.

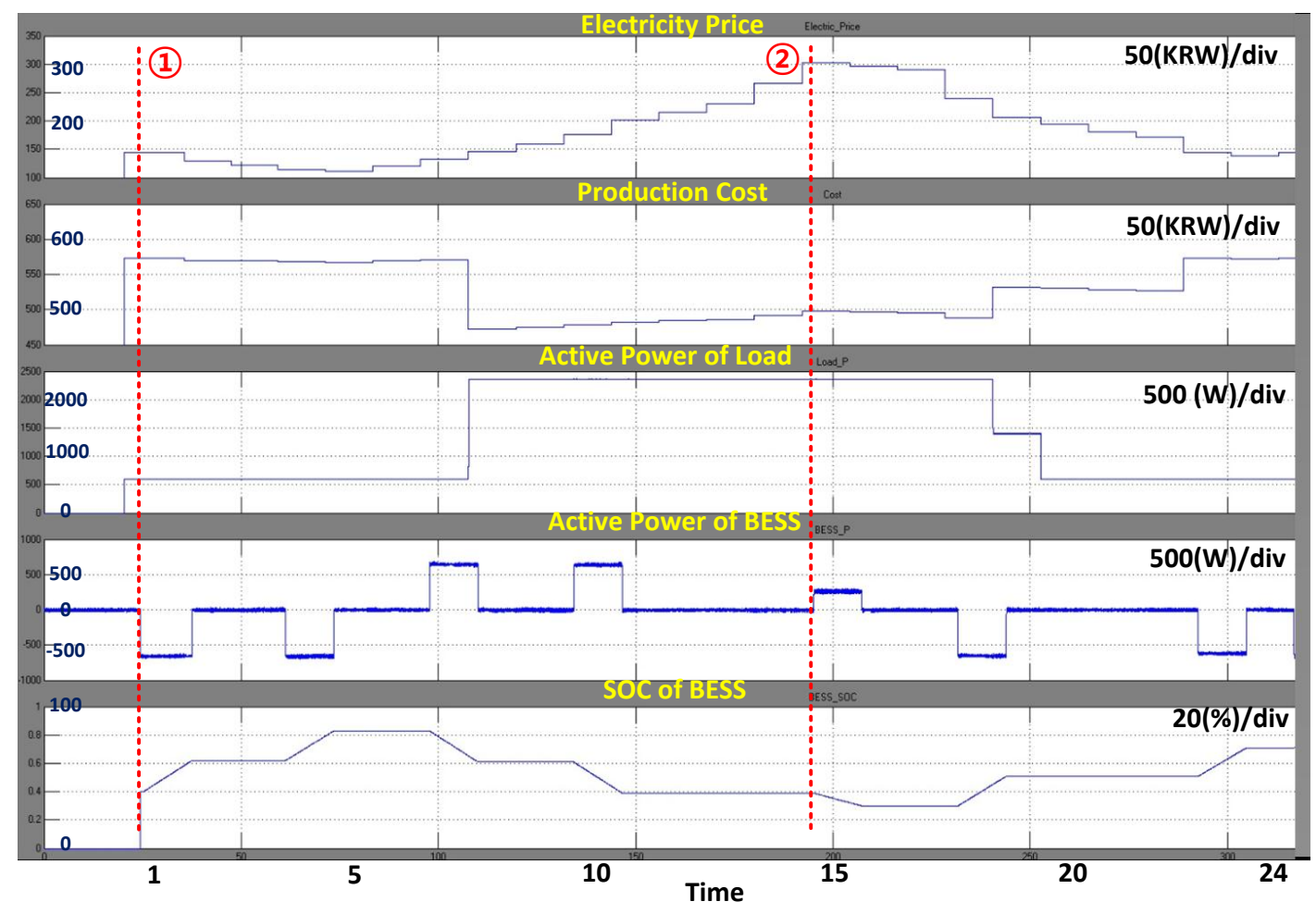

Figure 9. HILS test result of scenario 1.

\subsubsection{Scenario 2: DAP of Summer with EDR Signal (No Delay Signal)}

Figure 10 presents the HILS test result for DAP in summer with the EDR signal. This scenario is based on the scheduling result of the summer DA_RTP, and therefore it is the same as scenario 1 until the EDR signal is generated at (2) In this scenario, however, the EDR signal with the EDR occurred time and participation incentive is entered as seen in Figure 11, and this signal is sent to each agent by clicking on the EDR button in the MGCC program between (1) and (2) in Figure 10. When each 
agent receives the EDR signal, the agent executes a real-time algorithm for the EDR situation. The EDR time period is defined between two hours from the input time in the MGCC program. In this scenario, the time at 13 of EDR time (2) and $750 \mathrm{won} / \mathrm{kWh}$ for the incentive are entered. From Figure 10, the load reduces the power, and the BESS discharges at this EDR time period. If EDR has occurred, the BESS SOC is set to $20 \%$ lower than the usual day. The BESS is charged again at time 18 (3) at a low electricity price and SOC. The BESS does not charge at time 15 because EDR has finished as the electricity price is too high for charging. Finally, the BESS charges at times 21 and 24 because the electricity price is low. The load must reproduce for the reduced volume during the EDR period. Therefore, the load is shifted with 19 to $21 \mathrm{~h}$ having a low cost after the EDR period.

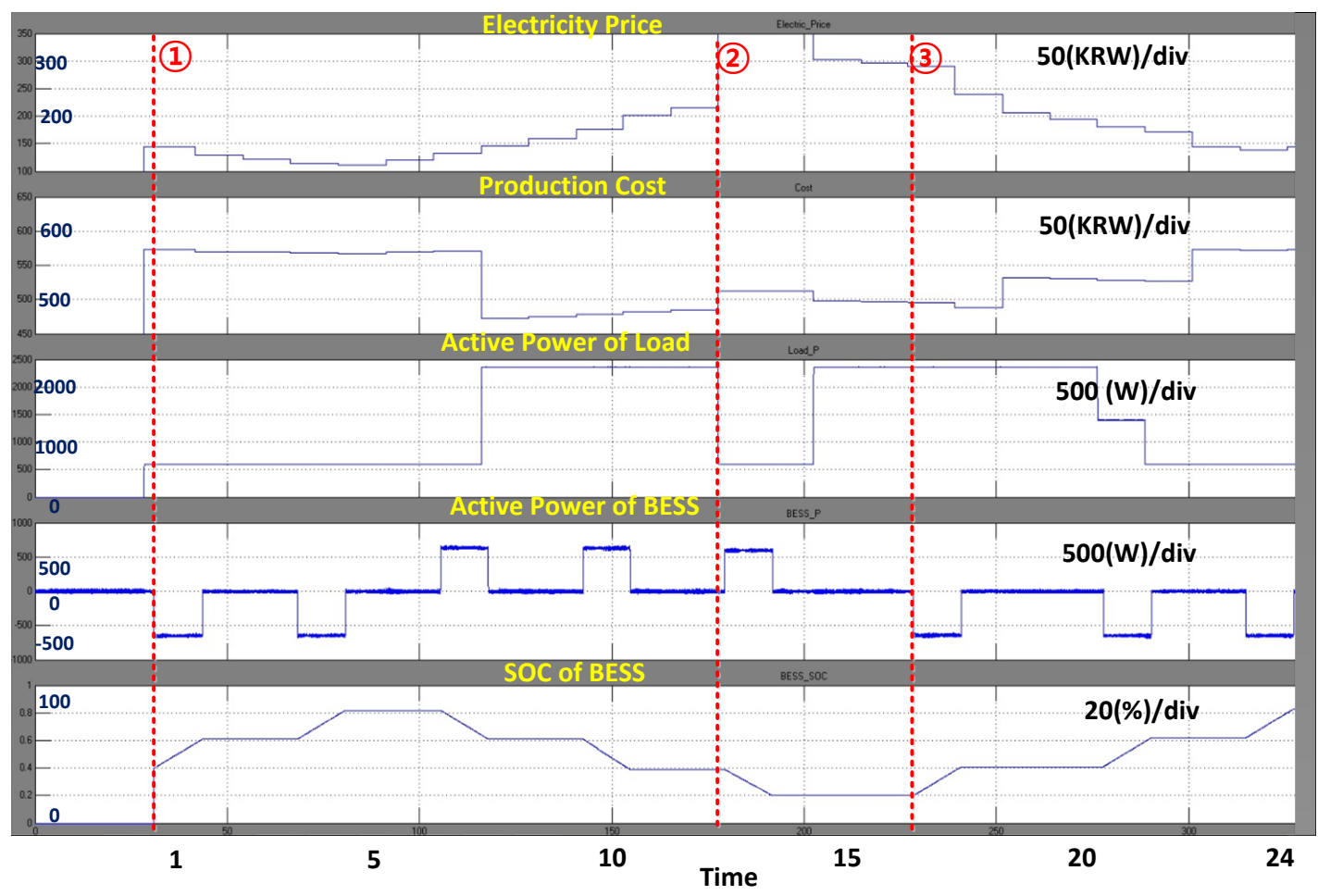

Figure 10. HILS test result of scenario 2.

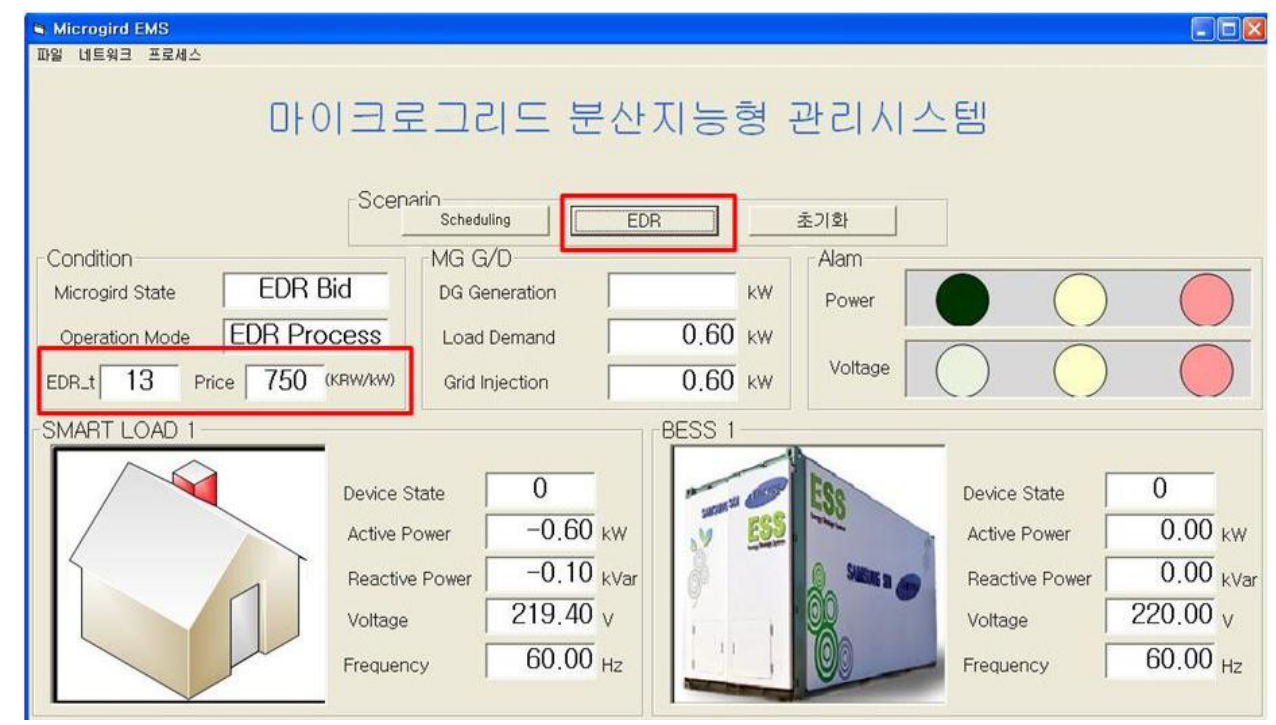

Figure 11. Emergency demand response (EDR) data input display in the MGCC program. 


\subsubsection{Scenario 3: DAP of Summer with the EDR Signal (No Delay Signal)}

In this scenario, as shown in Figure 12, most of the settings are the same as in scenario 2 except for the charging delay signal. The MGCC receives the BESS output after the EDR period. Therefore, it is possible to generate a charging delay signal as determined by the microgrid stability. In a grid-connected microgrid, all agents can operate for their own economic advantages under any situation. If the maximum allowable power is available for the transformer capacity, transmission, and distribution cable capacity at the PCC point, which is connected to the grid, the MGCC cannot allow the output of each agent from the standpoint of the global objective of the system. In this study, the maximum allowable power at the PCC is $2.5 \mathrm{~kW}$. The load shifting time is the same as in scenario 2. Therefore, the BESS charging time is delayed at $21 \mathrm{~h}$, which is in contrast to scenario 2 because the load is reduced at $21 \mathrm{~h}$. If the BESS charge at $21 \mathrm{~h}$, the microgrid's injection power does not exceed $2.5 \mathrm{~kW}$ at PCC point. So the MGCC does not generate a delay signal for BESS agent. Since then, the BESS changes a standby state at $22 \mathrm{~h}$ because the SOC is higher than $40 \%$ and maintains stable range, and the BESS charges by low electricity prices from $23 \mathrm{~h}$.

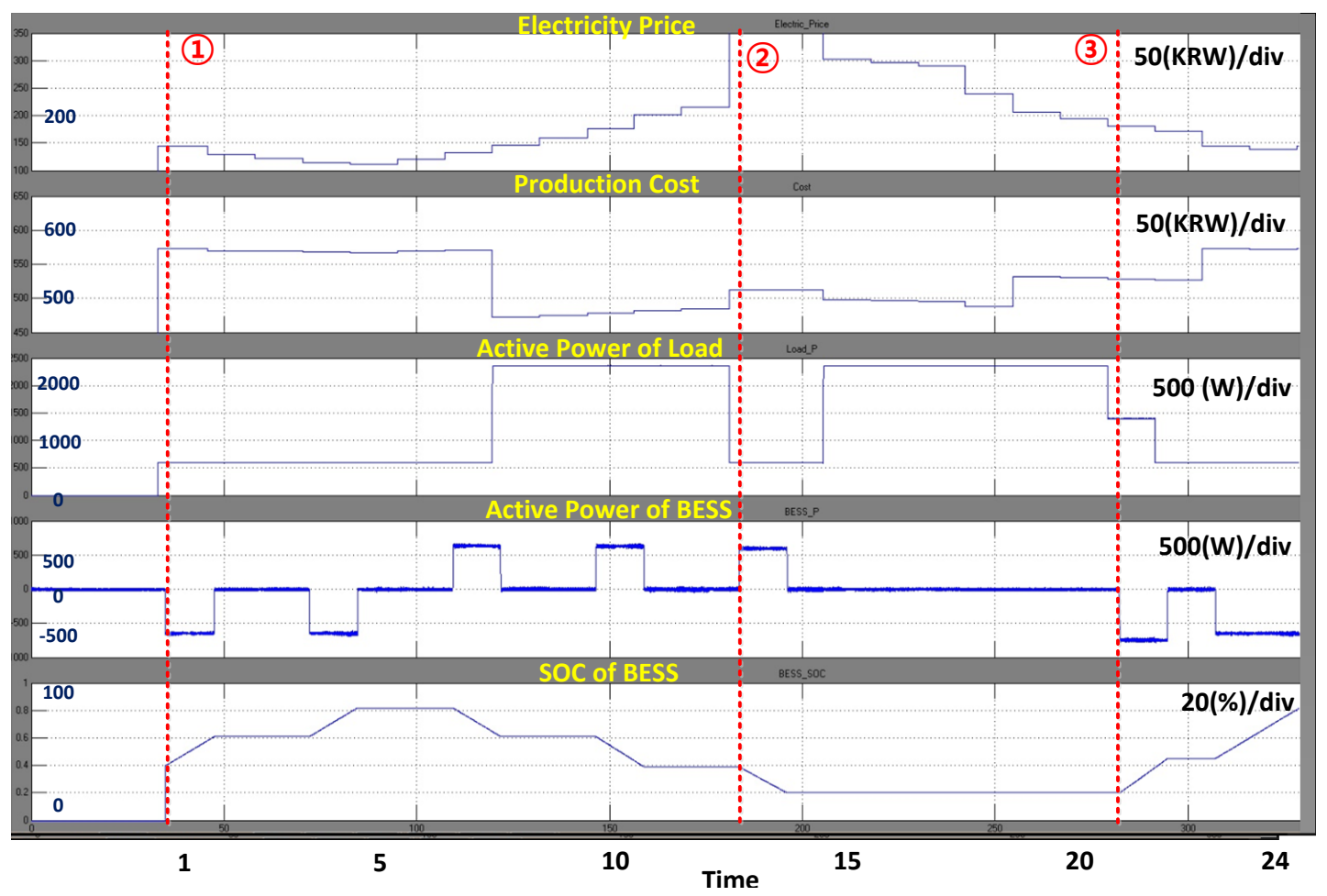

Figure 12. HILS test result of scenario 3.

\section{Experimental Results}

This study simulated the intelligent distributed operation and control in the smartgrid environment that the new distribution simulator connects the BESS and the intelligent load. The new distribution simulator can monitor the state of microgrid and electricity quality from a remote PC connected to the Ethernet. The new distribution simulator can construct any power system network as setting in software installed in the PC and store real-time data from the installed power quality meter. On the other hand, because there is no feature that can see the graphically data display stored in the program, it can be compared graphically using the Excel program This study simulated intelligent distributed operation and control in a smart grid environment in which the new distribution simulator connects the BESS and the intelligent load. The new distribution simulator can monitor the state of the microgrid and the electricity quality from a remote PC connected to the 
Ethernet. The new distribution simulator can construct any power system network with suitable settings in software installed in the PC and store real-time data from the installed power quality meter. However, the program is unable to display stored data graphically. MS Excel can be used for a graphical comparison.

Figure 13 shows a distribution simulator for hardware test. Figure 14 shows an intelligent load device and an energy storage device used in the hardware test. The two devices are connected to the new distribution simulator, and each device operates on the output from the connected agent. The inverter connected to the BESS and intelligent load has a rated capacity of $5 \mathrm{~kW}$. The oscilloscope is installed at the point where the hardware is connected to the simulator for comparison with the PQM data in the software.

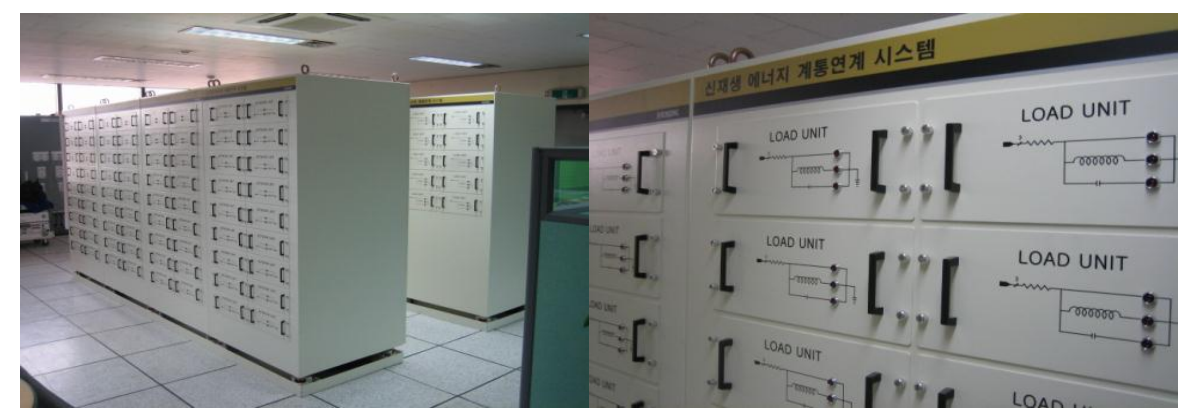

Figure 13. New distribution simulator in the laboratory.

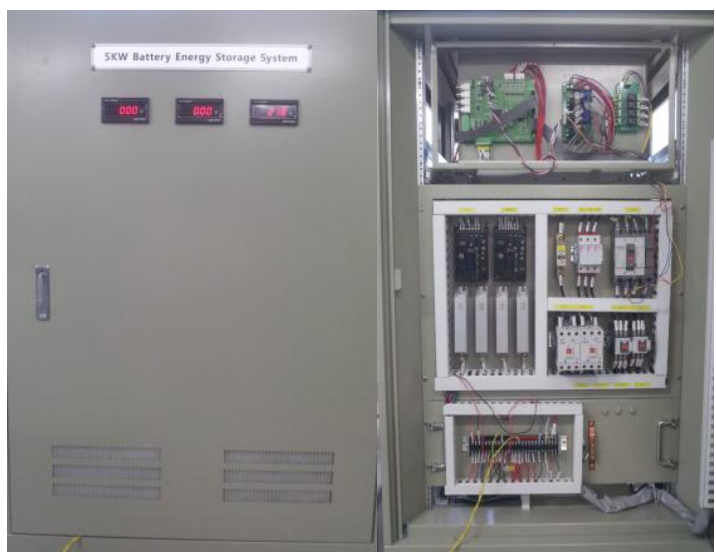

(a)

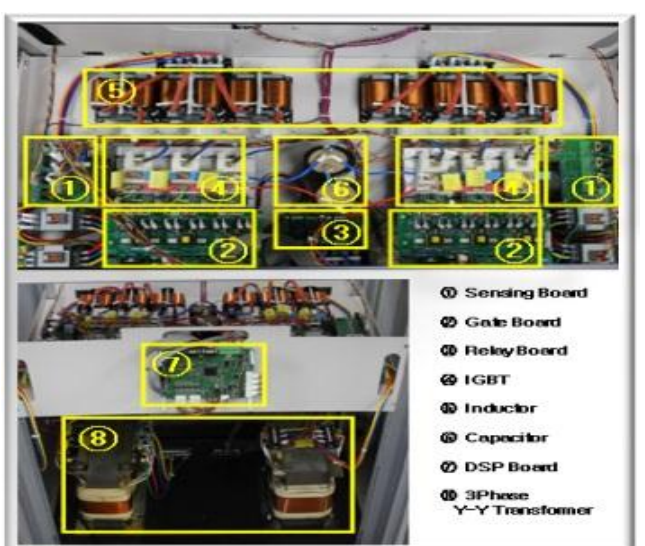

(b)

Figure 14. Hardware for experiments (a) 5 kW BESS; (b) 5 kW Load.

In this study, the hardware test was performed for scenario 1 and compared with the HILS test result. In the hardware test, it was difficult to visualize the electrical price and production costs, and the BESS SOC was calculated in the agent because of the simulation difference between the actual performance time of the BESS and the scaled time interval for simulation.

Figure 15 presents the HILS test result and the hardware test result for scenario 1 . The two test results differ, but the overall charging and discharging and load patterns are the same. However, because of the difference between the scaled time interval and the actual time, a few milliseconds delay occurred owing to data transmission and calculation of the algorithm. If the system operates for one day, this problem can be solved by synchronization using a GPS system and other algorithms for solving the time delay problem. 


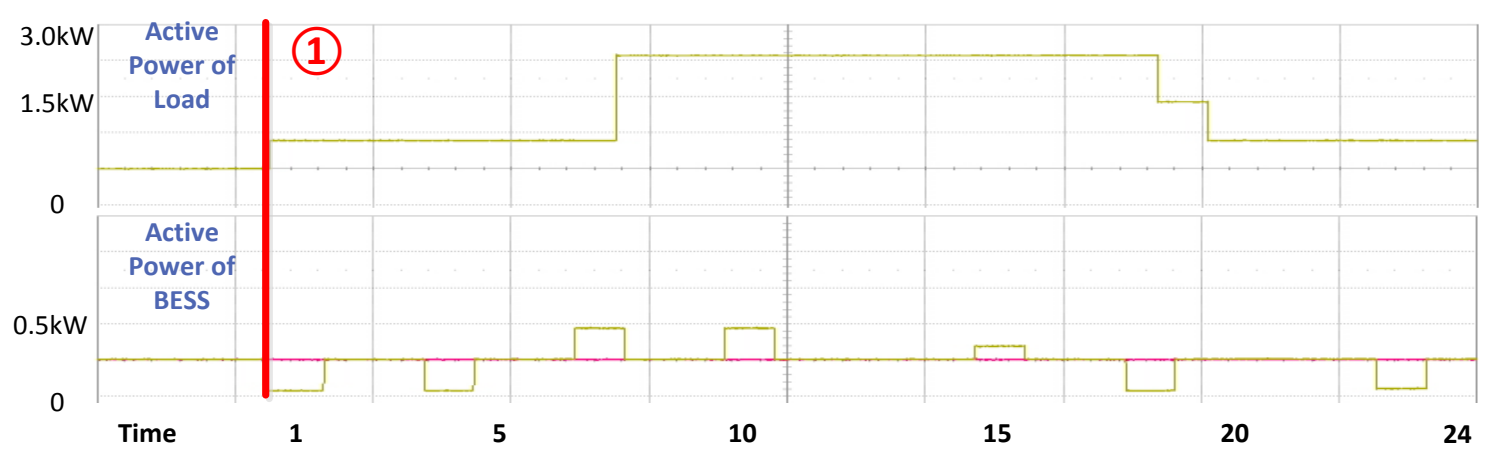

(a)

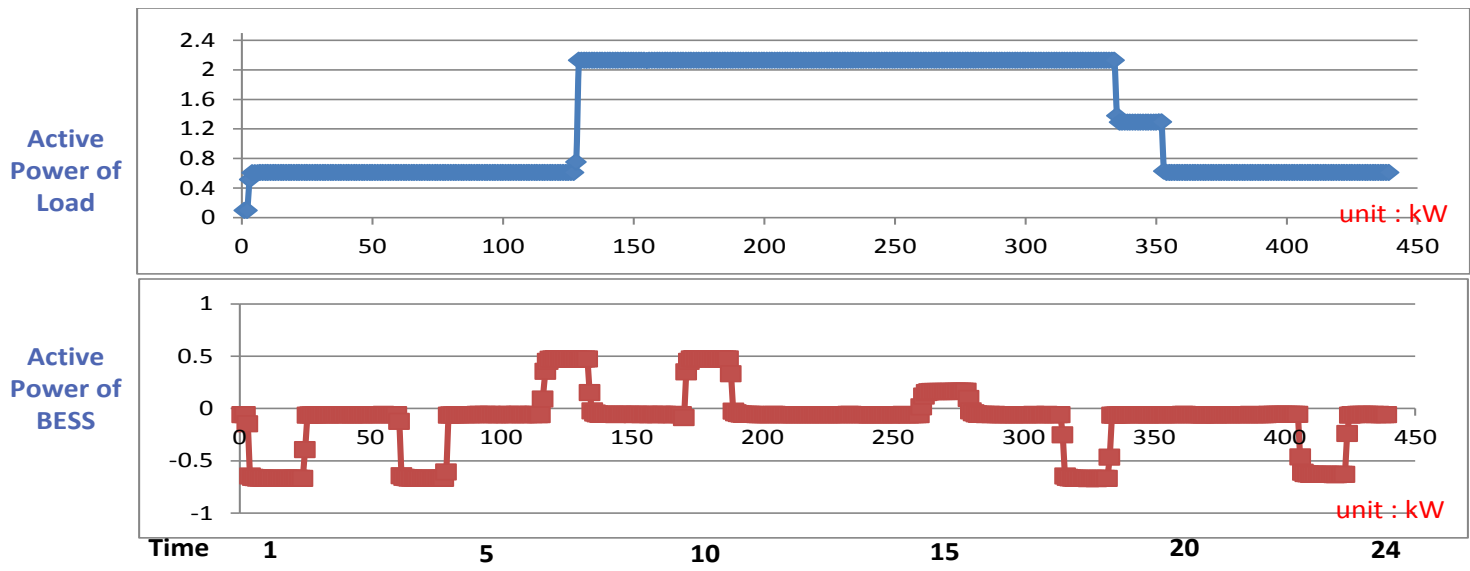

(b)

Figure 15. Hardware test result compared to the HILS test result of scenario 1 (a) Measurement at the oscilloscope installed in front of each piece of hardware; (b) Data stored in a new distribution simulator.

\section{Conclusions}

This study examined the microgrid operation method using MAS. The agent, through a micro control unit and microgrid components, is constructed for a hardware test, and an operation algorithm is included for DR. The operation algorithm includes a scheduling algorithm and a real-time operation algorithm for emergency situations. The load agent schedules with the objective of minimizing the production cost by considering hourly electricity prices and labor costs. The BESS agent schedules by using a fuzzy algorithm. Because the BESS operation algorithm considers the hourly BESS SOC and the electricity price, the BESS maintains a constant SOC range to prepare for emergency situations.

Each agent in the microgrid operates for its individual benefit. The MGCC in the study does not control the output of the agent even if EDR occurs. However, although these individual operation strategies ensure the maximum benefit of each agent, they can be a problem from the system stability perspective because the agents do not have access to system information. Although the microgrid constructs distributed MAS, the MGCC's role as a coordinator is required for monitoring and sending a grid's signal and information to each agent.

Consequently, we verified that the microgrid system that includes each agent applied the proposed algorithms for the load and the BESS with the DR program. Further, this study not only simulates but also implements in HILS be employing a new distribution simulator through wireless communication. In a future study, agents corresponding to renewable sources such as a wind turbine will be included in this system. A more detailed scenario and operation strategy considering renewable energy sources will be developed. 
Acknowledgments: This work was supported by the INHA UNIVERSITY Research Grant. This research was supported by Basic Science Research Program through the National Research Foundation of Korea (NRF) funded by the Ministry of Education, Science and Technology (Grant no. NRF-2013R1A1A1012667).

Author Contributions: Hee-Jun Cha carried out the main research tasks and wrote the full manuscript and proposed the original idea, analyzed and verified the results and the whole manuscript. Sang-Hyuk Kim and Il-Yop Chung provided technical support to verify the proposed algorithm in simulation software. Dong-Jun Won and Byung-Mun Han validated and double-checked the proposed algorithm, the results, and the whole manuscript.

Conflicts of Interest: The authors declare no conflict of interest.

\section{References}

1. Lasseter, B. Microgrids: Distributed power generation. In Proceedings of the IEEE Power Engineer Society Winter Meeting, Columbus, OH, USA, 28 January-1 February 2001; pp. 146-149.

2. Lasseter, R.H. Microgrids. In Proceedings of the IEEE Power Engineer Society Winter Meeting, New York, NY, USA, 27-31 January 2002; pp. 305-308.

3. Olivares, D.E.; Cañizares, C.A.; Kazerani, M.; Mehrizi-Sani, A.; Etemadi, A.H.; Iravani, R.; Hajimiragha, A.H.; Gomis-Bellmunt, O.; Saeedifard, M.; Palma-Behnke, T.; et al. Trends in Microgrid Control. Proc. IEEE Tans. Smart Grid 2014, 5, 1905-1919. [CrossRef]

4. Logenthiran, T.; Srinivasan, D.; Khambadkone, A.M.; Aung, H.N. Multiagent System for Real-Time Operation of a Microgrid in Real-Time Digital Simulator. Proc. IEEE Tans. Smart Grid 2012, 2, $925-933$. [CrossRef]

5. Dimeas, A.L.; Hatziargyriou, N.D. Operation of a Multiagent System for Microgrid Control. Proc. IEEE Trans. Power Syst. 2005, 3, 1447-1455. [CrossRef]

6. Dou, C.; Liu, B. Multi-Agent Based Hierarchical Hybrid Control for Smart Microgrid. Proc. IEEE Tans. Smart Grid 2013, 2, 771-778. [CrossRef]

7. Colson, C.M.; Nehrir, M.H. Comprehensive Real-Time Microgrid Power Management and Control with Distributed Agents. Proc. IEEE Tans. Smart Grid 2013, 4, 617-627. [CrossRef]

8. Braithwait, S.; Eakin, K. The Role of Demand Response in Electric Power Market Design; Laurits, R., Ed.; Christensen Associates, Inc.: Madison, WI, USA, 2002.

9. Conti, S.; Nicolosi, R.; Rizzo, S.A. Optimal dispatching of distributed generators in an MV autonomous micro-grid to minimize operating costs and emissions. In Proceeding of the IEEE International Symposium in Industrial Electronics (ISIE), Bari, Italy, 4-7 July 2010; pp. 2542-2547.

10. Colson, C.; Nehrir, M.; Poumousavi, S. Towards real-time microgrid power management using computational intelligence methods. In Proceeding of the IEEE Power and Energy General Meeting, Minneapolis, MN, USA, 1-8 July 2010.

11. Hee-Jun, C.; Jin-Young, C.; Dong-Jun, W. Smart load management in demand response using microgrid EMS. In Proceedings of the 2014 IEEE International Energy Conference, Cavtat, Croatia, 13-16 May 2014; pp. 833-837.

12. Suazo-Martinez, C.; Pereira-Bonvallet, E.; Palma-Behnke, R.; Zhang, X.-P. Impacts of Energy Storage on Short Term Operation Planning Under Centralized Spot Markets. Proc. IEEE Tans. Smart Grid 2014, 2, 1110-1118. [CrossRef]

13. Vazquez, S.; Lukic, S.M.; Galvan, E.; Franquelo, L.G.; Carrasco, J.M. Energy Storage Systems for Transport and Grid Applications. Proc. IEEE Tans. Ind. Electron. 2010, 12, 3881-3895. [CrossRef]

14. Negnevitsky, M. Artificial Intelligence, 2nd ed.; Hanbit Media: Seoul, Korea, 2011; pp. 128-171.

15. Oh, S.-J.; Yoo, C.-H.; Chung, I.-Y.; Won, D.-J. Hardware-in-the-Loop Simulation of Distributed Intelligent Energy Management System for Microgrids. Energies 2013, 6, 3263-3283. [CrossRef]

(C) 2015 by the authors; licensee MDPI, Basel, Switzerland. This article is an open access article distributed under the terms and conditions of the Creative Commons by Attribution (CC-BY) license (http://creativecommons.org/licenses/by/4.0/). 